\title{
Fubini's Theorem for Non-Negative or Non-Positive Functions
}

\author{
Noboru Endou \\ National Institute of Technology, Gifu College \\ 2236-2 Kamimakuwa, Motosu, Gifu, Japan
}

\begin{abstract}
Summary. The goal of this article is to show Fubini's theorem for nonnegative or non-positive measurable functions [10], 2], 3], using the Mizar system 1], 9]. We formalized Fubini's theorem in our previous article [5], but in that case we showed the Fubini's theorem for measurable sets and it was not enough as the integral does not appear explicitly.

On the other hand, the theorems obtained in this paper are more general and it can be easily extended to a general integrable function. Furthermore, it also can be easy to extend to functional space $L^{p}$ 12. It should be mentioned also that Hölzl and Heller [1] have introduced the Lebesgue integration theory in Isabelle/HOL and have proved Fubini's theorem there.
\end{abstract}

MSC: 28A35 03B35

Keywords: Fubini's theorem; extended real-valued non-negative (or non-positive) measurable function

MML identifier: MESFUN12, version: 8.1.07 5.47.1318

\section{Extended Real-Valued Characteristic Function}

Let $A, X$ be sets and $e$ be an extended real. The functor $\chi_{e, A, X}$ yielding a function from $X$ into $\overline{\mathbb{R}}$ is defined by

(Def. 1) for every object $x$ such that $x \in X$ holds if $x \in A$, then it $(x)=e$ and if $x \notin A$, then $i t(x)=0$.

Now we state the propositions:

(1) Let us consider a non empty set $X$, a set $A$, and a real number $r$. Then $r \cdot \chi_{A, X}=\chi_{r, A, X}$. 
(2) Let us consider a non empty set $X$, and a set $A$. Then

(i) $\chi_{+\infty, A, X}=\bar{\chi}_{A, X}$, and

(ii) $\chi_{-\infty, A, X}=-\bar{\chi}_{A, X}$.

(3) Let us consider sets $X, A$. Then $\chi_{A, X}$ is without $+\infty$ and without $-\infty$.

(4) Let us consider a non empty set $X$, a set $A$, and a real number $r$. Then

(i) $\operatorname{rng} \chi_{r, A, X} \subseteq\{0, r\}$, and

(ii) $\chi_{r, A, X}$ is without $+\infty$ and without $-\infty$.

The theorem is a consequence of (3) and (1).

(5) Let us consider a non empty set $X$, a $\sigma$-field $S$ of subsets of $X$, a non empty partial function $f$ from $X$ to $\overline{\mathbb{R}}$, and a $\sigma$-measure $M$ on $S$. Suppose $f$ is simple function in $S$. Then there exists a non empty finite sequence $E$ of separated subsets of $S$ and there exists a finite sequence $a$ of elements of $\overline{\mathbb{R}}$ and there exists a finite sequence $r$ of elements of $\mathbb{R}$ such that $E$ and $a$ are representation of $f$ and for every natural number $n, a(n)=r(n)$ and $f\left\lceil E(n)=\chi_{r(n), E(n), X}\lceil E(n)\right.$ and if $E(n)=\emptyset$, then $r(n)=0$.

Proof: Consider $E$ being a finite sequence of separated subsets of $S, b$ being a finite sequence of elements of $\overline{\mathbb{R}}$ such that $E$ and $b$ are representation of $f$. For every natural number $n$ such that $E(n) \neq \emptyset$ holds $b(n) \in \mathbb{R}$ by [8, (32)]. Define $\mathcal{Q}\left[\right.$ natural number, object] $\equiv$ if $E\left(\$_{1}\right) \neq \emptyset$, then $\$_{2}=b\left(\$_{1}\right)$ and if $E\left(\$_{1}\right)=\emptyset$, then $\$_{2}=0$. For every natural number $n$ such that $n \in$ Seg len $E$ there exists an element $a$ of $\overline{\mathbb{R}}$ such that $\mathcal{Q}[n, a]$. Consider $a$ being a finite sequence of elements of $\overline{\mathbb{R}}$ such that $\operatorname{dom} a=\operatorname{Seg} \operatorname{len} E$ and for every natural number $n$ such that $n \in \operatorname{Seg}$ len $E$ holds $\mathcal{Q}[n, a(n)]$. Define $\mathcal{R}$ [natural number, object] $\equiv \$_{2}=a\left(\$_{1}\right)$. For every natural number $n$ such that $n \in$ Seg len $E$ there exists an element $r$ of $\mathbb{R}$ such that $\mathcal{R}[n, r]$. Consider $r$ being a finite sequence of elements of $\mathbb{R}$ such that $\operatorname{dom} r=\operatorname{Seg}$ len $E$ and for every natural number $n$ such that $n \in \operatorname{Seg}$ len $E$ holds $\mathcal{R}[n, r(n)]$. For every natural number $n$ such that $n \in \operatorname{dom} E$ for every object $x$ such that $x \in E(n)$ holds $f(x)=a(n)$. For every natural number $n, a(n)=r(n)$ and $f\left\lceil E(n)=\chi_{r(n), E(n), X}\lceil E(n)\right.$ and if $E(n)=\emptyset$, then $r(n)=0$.

Let $F$ be a finite sequence-like function. Let us observe that $F$ is disjoint valued if and only if the condition (Def. 2) is satisfied.

(Def. 2) for every natural numbers $m, n$ such that $m, n \in \operatorname{dom} F$ and $m \neq n$ holds $F(m)$ misses $F(n)$.

Now we state the propositions: 
(6) Let us consider a non empty set $X$, a $\sigma$-field $S$ of subsets of $X$, and elements $E_{1}, E_{2}$ of $S$. Suppose $E_{1}$ misses $E_{2}$. Then $\left\langle E_{1}, E_{2}\right\rangle$ is a finite sequence of separated subsets of $S$.

(7) Let us consider a non empty set $X$, subsets $A_{1}, A_{2}$ of $X$, and real numbers $r_{1}, r_{2}$. Then $\left\langle\chi_{r_{1}, A_{1}, X}, \chi_{r_{2}, A_{2}, X}\right\rangle$ is a summable finite sequence of elements of $\overline{\mathbb{R}}^{X}$. The theorem is a consequence of (4).

(8) Let us consider a non empty set $X$, and a summable finite sequence $F$ of elements of $\overline{\mathbb{R}}^{X}$. Suppose len $F \geqslant 2$. Then $\left(\left(\sum_{\alpha=0}^{\kappa} F(\alpha)\right)_{\kappa \in \mathbb{N}}\right)_{/ 2}=F_{/ 1}+F_{/ 2}$.

(9) Let us consider a non empty set $X$, and a function $f$ from $X$ into $\overline{\mathbb{R}}$. Then $f+\left(X \longmapsto 0_{\overline{\mathbb{R}}}\right)=f$.

(10) Let us consider a non empty set $X$, and a summable finite sequence $F$ of elements of $\overline{\mathbb{R}}^{X}$. Then

(i) $\operatorname{dom} F=\operatorname{dom}\left(\sum_{\alpha=0}^{\kappa} F(\alpha)\right)_{\kappa \in \mathbb{N}}$, and

(ii) for every natural number $n$ such that $n \in \operatorname{dom} F$ holds $\left(\left(\sum_{\alpha=0}^{\kappa} F(\alpha)\right)_{\kappa \in \mathbb{N}}\right)_{/ n}=\left(\sum_{\alpha=0}^{\kappa} F(\alpha)\right)_{\kappa \in \mathbb{N}}(n)$, and

(iii) for every natural number $n$ and for every element $x$ of $X$ such that $1 \leqslant$ $n<\operatorname{len} F$ holds $\left(\left(\sum_{\alpha=0}^{\kappa} F(\alpha)\right)_{\kappa \in \mathbb{N}}\right)_{/ n+1}(x)=\left(\left(\sum_{\alpha=0}^{\kappa} F(\alpha)\right)_{\kappa \in \mathbb{N}}\right)_{/ n}(x)+$ $F_{/ n+1}(x)$.

Proof: For every natural number $n$ and for every element $x$ of $X$ such that $1 \leqslant n<\operatorname{len} F$ holds $\left(\left(\sum_{\alpha=0}^{\kappa} F(\alpha)\right)_{\kappa \in \mathbb{N}}\right)_{/ n+1}(x)=\left(\left(\sum_{\alpha=0}^{\kappa} F(\alpha)\right)_{\kappa \in \mathbb{N}}\right)_{/ n}(x)+$ $F_{/ n+1}(x)$.

(11) Let us consider a non empty set $X$, a $\sigma$-field $S$ of subsets of $X$, a function $f$ from $X$ into $\overline{\mathbb{R}}$, a finite sequence $E$ of separated subsets of $S$, and a summable finite sequence $F$ of elements of $\overline{\mathbb{R}}^{X}$. Suppose $\operatorname{dom} E=\operatorname{dom} F$ and $\operatorname{dom} f=\bigcup \operatorname{rng} E$ and for every natural number $n$ such that $n \in$ $\operatorname{dom} F$ there exists a real number $r$ such that $F_{/ n}=r \cdot \chi_{E(n), X}$ and $f=$ $\left(\left(\sum_{\alpha=0}^{\kappa} F(\alpha)\right)_{\kappa \in \mathbb{N}}\right) /$ len $F$. Then

(i) for every element $x$ of $X$ and for every natural numbers $m, n$ such that $m, n \in \operatorname{dom} F$ and $x \in E(m)$ and $m \neq n$ holds $F_{/ n}(x)=0$, and

(ii) for every element $x$ of $X$ and for every natural numbers $m, n$ such that $m, n \in \operatorname{dom} F$ and $x \in E(m)$ and $n<m$ holds $\left(\left(\sum_{\alpha=0}^{\kappa} F(\alpha)\right)_{\kappa \in \mathbb{N}}\right)_{/ n}(x)=0$, and

(iii) for every element $x$ of $X$ and for every natural numbers $m, n$ such that $m, n \in \operatorname{dom} F$ and $x \in E(m)$ and $n \geqslant m$ holds $\left(\left(\sum_{\alpha=0}^{\kappa} F(\alpha)\right)_{\kappa \in \mathbb{N}}\right)_{/ n}(x)=f(x)$, and

(iv) for every element $x$ of $X$ and for every natural number $m$ such that $m \in \operatorname{dom} F$ and $x \in E(m)$ holds $F_{/ m}(x)=f(x)$, and 
(v) $f$ is simple function in $S$.

Proof: For every element $x$ of $X$ and for every natural numbers $m, n$ such that $m, n \in \operatorname{dom} F$ and $x \in E(m)$ and $m \neq n$ holds $F_{/ n}(x)=0$. For every element $x$ of $X$ and for every natural numbers $m, n$ such that $m$, $n \in \operatorname{dom} F$ and $x \in E(m)$ and $n<m$ holds $\left(\left(\sum_{\alpha=0}^{\kappa} F(\alpha)\right)_{\kappa \in \mathbb{N}}\right)_{/ n}(x)=0$. For every element $x$ of $X$ and for every natural numbers $m, n$ such that $m, n \in \operatorname{dom} F$ and $x \in E(m)$ and $n \geqslant m$ holds $\left(\left(\sum_{\alpha=0}^{\kappa} F(\alpha)\right)_{\kappa \in \mathbb{N}}\right) / n(x)=$ $f(x)$. For every element $x$ of $X$ and for every natural number $m$ such that $m \in \operatorname{dom} F$ and $x \in E(m)$ holds $F_{/ m}(x)=f(x)$. For every element $x$ of $X$ such that $x \in \operatorname{dom} f$ holds $|f(x)|<+\infty$ by [7, (41)]. For every natural number $n$ and for every elements $x, y$ of $X$ such that $n \in \operatorname{dom} E$ and $x$, $y \in E(n)$ holds $f(x)=f(y)$.

(12) Let us consider a non empty set $X$, a $\sigma$-field $S$ of subsets of $X$, a $\sigma$ measure $M$ on $S$, and an element $E$ of $S$. Then $\chi_{E, X}$ is simple function in $S$.

Proof: Reconsider $E_{2}=X \backslash E$ as an element of $S$. Reconsider $E_{3}=\langle E$, $\left.E_{2}\right\rangle$ as a finite sequence of separated subsets of $S .1 \cdot \chi_{E, X}=\chi_{1, E, X}$ and $0 \cdot \chi_{E_{2}, X}=\chi_{0, E_{2}, X}$. Reconsider $F=\left\langle 1 \cdot \chi_{E, X}, 0 \cdot \chi_{E_{2}, X}\right\rangle$ as a summable finite sequence of elements of $\overline{\mathbb{R}}^{X}$. For every natural number $n$ such that $n \in \operatorname{dom} F$ there exists a real number $r$ such that $F_{/ n}=r \cdot \chi_{E_{3}(n), X}$. $\left(\left(\sum_{\alpha=0}^{\kappa} F(\alpha)\right)_{\kappa \in \mathbb{N}}\right)_{/ \operatorname{len} F}=F_{/ 1}+F_{/ 2} \cdot\left(\left(\sum_{\alpha=0}^{\kappa} F(\alpha)\right)_{\kappa \in \mathbb{N}}\right)_{/ \text {len } F}=\chi_{E, X}$.

(13) Let us consider a non empty set $X$, a $\sigma$-field $S$ of subsets of $X$, a $\sigma$ measure $M$ on $S$, elements $A, B$ of $S$, and an extended real $e$. Then $\chi_{e, A, X}$ is measurable on $B$. The theorem is a consequence of (2) and (1).

(14) Let us consider a set $X$, subsets $A_{1}, A_{2}$ of $X$, and an extended real $e$. Then $\chi_{e, A_{1}, X}\left\lceil A_{2}=\chi_{e, A_{1} \cap A_{2}, X}\left\lceil A_{2}\right.\right.$.

(15) Let us consider a non empty set $X$, a $\sigma$-field $S$ of subsets of $X$, a $\sigma$ measure $M$ on $S$, elements $A, B, C$ of $S$, and an extended real $e$. If $C \subseteq B$, then $\chi_{e, A, X}\lceil B$ is measurable on $C$. The theorem is a consequence of (13).

(16) Let us consider a set $X$, subsets $A_{1}, A_{2}$ of $X$, an extended real $e$, and an object $x$. If $A_{1}$ misses $A_{2}$, then $\left(\chi_{e, A_{1}, X}\left\lceil A_{2}\right)(x)=0\right.$.

(17) Let us consider a set $X$, a subset $A$ of $X$, and an extended real $e$. Then

(i) if $e \geqslant 0$, then $\chi_{e, A, X}$ is non-negative, and

(ii) if $e \leqslant 0$, then $\chi_{e, A, X}$ is non-positive.

(18) Let us consider sets $A, X$, and a subset $B$ of $X$. Then $\operatorname{dom}\left(\chi_{A, X}\lceil B)=B\right.$. 


\section{Some Properties of Integration}

Now we state the propositions:

(19) Let us consider a non empty set $X$, a $\sigma$-field $S$ of subsets of $X$, and a partial function $f$ from $X$ to $\overline{\mathbb{R}}$. If $f$ is empty, then $f$ is simple function in $S$.

Proof: Reconsider $E_{4}=\emptyset$ as an element of $S$. Reconsider $F=\left\langle E_{4}\right\rangle$ as a finite sequence of separated subsets of $S$. For every natural number $n$ and for every elements $x, y$ of $X$ such that $n \in \operatorname{dom} F$ and $x, y \in F(n)$ holds $f(x)=f(y)$.

(20) Let us consider a non empty set $X$, a $\sigma$-field $S$ of subsets of $X$, a $\sigma$ measure $M$ on $S$, and elements $E_{1}, E_{2}$ of $S$. Then $\int \chi_{E_{1}, X}\left\lceil E_{2} \mathrm{~d} M=\right.$ $M\left(E_{1} \cap E_{2}\right)$.

Let us consider a non empty set $X$, a $\sigma$-field $S$ of subsets of $X$, a $\sigma$-measure $M$ on $S$, elements $E_{1}, E_{2}$ of $S$, and partial functions $f, g$ from $X$ to $\overline{\mathbb{R}}$. Now we state the propositions:

(21) Suppose $E_{1}=\operatorname{dom} f$ and $f$ is non-negative and $f$ is measurable on $E_{1}$ and $E_{2}=\operatorname{dom} g$ and $g$ is non-negative and $g$ is measurable on $E_{2}$. Then $\int f+g \mathrm{~d} M=\int f \uparrow \operatorname{dom}(f+g) \mathrm{d} M+\int g \uparrow \operatorname{dom}(f+g) \mathrm{d} M$.

(22) Suppose $E_{1}=\operatorname{dom} f$ and $f$ is non-positive and $f$ is measurable on $E_{1}$ and $E_{2}=\operatorname{dom} g$ and $g$ is non-positive and $g$ is measurable on $E_{2}$. Then $\int f+g \mathrm{~d} M=\int f\left\lceil\operatorname{dom}(f+g) \mathrm{d} M+\int g\lceil\operatorname{dom}(f+g) \mathrm{d} M\right.$. The theorem is a consequence of (21).

(23) Suppose $E_{1}=\operatorname{dom} f$ and $f$ is non-negative and $f$ is measurable on $E_{1}$ and $E_{2}=\operatorname{dom} g$ and $g$ is non-positive and $g$ is measurable on $E_{2}$. Then

(i) $\int f-g \mathrm{~d} M=\int f\left\lceil\operatorname{dom}(f-g) \mathrm{d} M-\int g\lceil\operatorname{dom}(f-g) \mathrm{d} M\right.$, and

(ii) $\int g-f \mathrm{~d} M=\int g \uparrow \operatorname{dom}(g-f) \mathrm{d} M-\int f \uparrow \operatorname{dom}(g-f) \mathrm{d} M$.

The theorem is a consequence of (21).

(24) Let us consider a non empty set $X$, a $\sigma$-field $S$ of subsets of $X$, a $\sigma$ measure $M$ on $S$, an element $E$ of $S$, a partial function $f$ from $X$ to $\overline{\mathbb{R}}$, and a real number $r$. Suppose $E=\operatorname{dom} f$ and $f$ is non-positive or non-negative and $f$ is measurable on $E$. Then $\int r \cdot f \mathrm{~d} M=r \cdot \int f \mathrm{~d} M$. 


\section{Sections of Partial Function}

Now we state the proposition:

(25) Let us consider non empty sets $X, Y$, an element $A$ of $2^{X \times Y}$, and sets $x, y$. Suppose $x \in X$ and $y \in Y$. Then

(i) $\langle x, y\rangle \in A$ iff $x \in \operatorname{Ysection}(A, y)$, and

(ii) $\langle x, y\rangle \in A$ iff $y \in \operatorname{Xsection}(A, x)$.

Let $X_{1}, X_{2}$ be non empty sets, $Y$ be a set, $f$ be a partial function from $X_{1} \times$ $X_{2}$ to $Y$, and $x$ be an element of $X_{1}$. The functor $\operatorname{ProjPMap1}(f, x)$ yielding a partial function from $X_{2}$ to $Y$ is defined by

(Def. 3) $\operatorname{dom} i t=X \operatorname{section}(\operatorname{dom} f, x)$ and for every element $y$ of $X_{2}$ such that $\langle x$, $y\rangle \in \operatorname{dom} f$ holds $i t(y)=f(x, y)$.

Let $y$ be an element of $X_{2}$. The functor ProjPMap2 $(f, y)$ yielding a partial function from $X_{1}$ to $Y$ is defined by

(Def. 4) $\operatorname{dom} i t=Y \operatorname{section}(\operatorname{dom} f, y)$ and for every element $x$ of $X_{1}$ such that $\langle x$, $y\rangle \in \operatorname{dom} f$ holds it $(x)=f(x, y)$.

Now we state the propositions:

(26) Let us consider non empty sets $X_{1}, X_{2}$, a set $Y$, a partial function $f$ from $X_{1} \times X_{2}$ to $Y$, an element $x$ of $X_{1}$, and an element $y$ of $X_{2}$. Then

(i) if $x \in \operatorname{dom} \operatorname{ProjPMap} 2(f, y)$, then $(\operatorname{ProjPMap} 2(f, y))(x)=f(x, y)$, and

(ii) if $y \in \operatorname{dom} \operatorname{ProjPMap} 1(f, x)$, then $\operatorname{ProjPMap} 1(f, x))(y)=f(x, y)$.

(27) Let us consider non empty sets $X_{1}, X_{2}, Y$, a function $f$ from $X_{1} \times X_{2}$ into $Y$, an element $x$ of $X_{1}$, and an element $y$ of $X_{2}$. Then

(i) $\operatorname{ProjPMap} 1(f, x)=\operatorname{curry}(f, x)$, and

(ii) $\operatorname{ProjPMap} 2(f, y)=\operatorname{curry}^{\prime}(f, y)$.

The theorem is a consequence of (26).

(28) Let us consider non empty sets $X, Y, Z$, a partial function $f$ from $X \times$ $Y$ to $Z$, an element $x$ of $X$, an element $y$ of $Y$, and a set $A$. Then

(i) $\operatorname{Xsection}\left(f^{-1}(A), x\right)=(\operatorname{ProjPMap} 1(f, x))^{-1}(A)$, and

(ii) $\operatorname{Ysection}\left(f^{-1}(A), y\right)=(\operatorname{ProjPMap} 2(f, y))^{-1}(A)$.

(29) Let us consider non empty sets $X_{1}, X_{2}$, an element $x$ of $X_{1}$, an element $y$ of $X_{2}$, a real number $r$, and a partial function $f$ from $X_{1} \times X_{2}$ to $\overline{\mathbb{R}}$. Then

(i) $\operatorname{ProjPMap} 1(r \cdot f, x)=r \cdot \operatorname{ProjPMap} 1(f, x)$, and 
(ii) $\operatorname{ProjPMap2}(r \cdot f, y)=r \cdot \operatorname{ProjPMap2}(f, y)$.

(30) Let us consider non empty sets $X_{1}, X_{2}$, a partial function $f$ from $X_{1} \times$ $X_{2}$ to $\overline{\mathbb{R}}$, an element $x$ of $X_{1}$, and an element $y$ of $X_{2}$. Suppose for every element $z$ of $X_{1} \times X_{2}$ such that $z \in \operatorname{dom} f$ holds $f(z)=0$. Then

(i) $(\operatorname{ProjPMap} 2(f, y))(x)=0$, and

(ii) $(\operatorname{ProjPMap} 1(f, x))(y)=0$.

(31) Let us consider non empty sets $X_{1}, X_{2}$, a $\sigma$-field $S_{1}$ of subsets of $X_{1}$, a $\sigma$-field $S_{2}$ of subsets of $X_{2}$, an element $x$ of $X_{1}$, an element $y$ of $X_{2}$, and a partial function $f$ from $X_{1} \times X_{2}$ to $\overline{\mathbb{R}}$. Suppose $f$ is simple function in $\sigma\left(\operatorname{MeasRect}\left(S_{1}, S_{2}\right)\right)$. Then

(i) ProjPMap1 $(f, x)$ is simple function in $S_{2}$, and

(ii) ProjPMap2 $(f, y)$ is simple function in $S_{1}$.

Proof: Consider $F$ being a finite sequence of separated subsets of $\sigma$ (Meas$\left.\operatorname{Rect}\left(S_{1}, S_{2}\right)\right)$ such that $\operatorname{dom} f=\bigcup \operatorname{rng} F$ and for every natural number $n$ and for every elements $z_{1}, z_{2}$ of $X_{1} \times X_{2}$ such that $n \in \operatorname{dom} F$ and $z_{1}, z_{2} \in F(n)$ holds $f\left(z_{1}\right)=f\left(z_{2}\right)$. Define $\mathcal{H}$ (natural number) $=$ MeasurableXsection $\left(F\left(\$_{1}\right), x\right)$. Consider $H$ being a finite sequence of elements of $S_{2}$ such that len $H=$ len $F$ and for every natural number $n$ such that $n \in \operatorname{dom} H$ holds $H(n)=\mathcal{H}(n)$. Reconsider $F_{1}=F$ as a finite sequence of elements of $2^{X_{1} \times X_{2}}$. Reconsider $F_{2}=H$ as a finite sequence of elements of $2^{X_{2}}$. For every natural number $n$ such that $n \in \operatorname{dom} F_{2}$ holds $F_{2}(n)=\mathrm{X} \operatorname{section}\left(F_{1}(n), x\right)$. For every natural number $n$ and for every elements $y_{1}, y_{2}$ of $X_{2}$ such that $n \in \operatorname{dom} H$ and $y_{1}, y_{2} \in H(n)$ holds $(\operatorname{ProjPMap} 1(f, x))\left(y_{1}\right)=(\operatorname{ProjPMap} 1(f, x))\left(y_{2}\right)$. Define $\mathcal{G}$ (natural number $)=$ MeasurableYsection $\left(F\left(\$_{1}\right), y\right)$. Consider $G$ being a finite sequence of elements of $S_{1}$ such that len $G=\operatorname{len} F$ and for every natural number $n$ such that $n \in \operatorname{dom} G$ holds $G(n)=\mathcal{G}(n)$. Reconsider $G_{1}=G$ as a finite sequence of elements of $2^{X_{1}}$. For every natural number $n$ such that $n \in \operatorname{dom} G_{1}$ holds $G_{1}(n)=\operatorname{Ysection}\left(F_{1}(n), y\right)$. For every natural number $n$ and for every elements $x_{1}, x_{2}$ of $X_{1}$ such that $n \in \operatorname{dom} G$ and $x_{1}, x_{2} \in G(n)$ holds (ProjPMap2 $\left.(f, y)\right)\left(x_{1}\right)=(\operatorname{ProjPMap} 2(f, y))\left(x_{2}\right)$.

Let us consider non empty sets $X_{1}, X_{2}$, an element $x$ of $X_{1}$, an element $y$ of $X_{2}$, and a partial function $f$ from $X_{1} \times X_{2}$ to $\overline{\mathbb{R}}$. Now we state the propositions:

(32) If $f$ is non-negative, then $\operatorname{ProjPMap} 1(f, x)$ is non-negative and ProjPMap2 $(f, y)$ is non-negative.

Proof: For every object $q$ such that $q \in \operatorname{dom} \operatorname{ProjPMap} 1(f, x)$ holds $0 \leqslant(\operatorname{ProjPMap} 1(f, x))(q)$. For every object $p$ such that $p \in \operatorname{dom}$ ProjPMap2 $(f, y)$ holds $0 \leqslant(\operatorname{ProjPMap} 2(f, y))(p)$. 
(33) If $f$ is non-positive, then $\operatorname{ProjPMap} 1(f, x)$ is non-positive and ProjPMap2 $(f, y)$ is non-positive.

Proof: For every set $q$ such that $q \in \operatorname{dom} \operatorname{ProjPMap} 1(f, x)$ holds $0 \geqslant$ $(\operatorname{ProjPMap} 1(f, x))(q)$. For every set $p$ such that $p \in \operatorname{dom} \operatorname{ProjPMap} 2(f, y)$ holds $0 \geqslant(\operatorname{ProjPMap} 2(f, y))(p)$ by [6, (8)].

(34) Let us consider non empty sets $X_{1}, X_{2}$, an element $x$ of $X_{1}$, an element $y$ of $X_{2}$, a subset $A$ of $X_{1} \times X_{2}$, and a partial function $f$ from $X_{1} \times X_{2}$ to $\overline{\mathbb{R}}$. Then

(i) $\operatorname{ProjPMap1}(f\lceil A, x)=\operatorname{ProjPMap} 1(f, x)\lceil\operatorname{Xsection}(A, x)$, and

(ii) $\operatorname{ProjPMap2}(f\lceil A, y)=\operatorname{ProjPMap} 2(f, y)\lceil\operatorname{Ysection}(A, y)$.

The theorem is a consequence of (25).

(35) Let us consider non empty sets $X_{1}, X_{2}$, a subset $A$ of $X_{1} \times X_{2}$, an element $x$ of $X_{1}$, and an element $y$ of $X_{2}$. Then

(i) $\operatorname{ProjPMap} 1\left(\bar{\chi}_{A, X_{1} \times X_{2}}, x\right)=\bar{\chi}_{\mathrm{Xsection}(A, x), X_{2}}$, and

(ii) ProjPMap2 $\left(\bar{\chi}_{A, X_{1} \times X_{2}}, y\right)=\bar{\chi}_{\operatorname{Ysection}(A, y), X_{1}}$.

The theorem is a consequence of (27) and (25).

(36) Let us consider a non empty set $X$, a $\sigma$-field $S$ of subsets of $X$, a $\sigma$ measure $M$ on $S$, partial functions $f, g$ from $X$ to $\overline{\mathbb{R}}$, and an element $E$ of $S$. Suppose $f\lceil E=g\lceil E$ and $E \subseteq \operatorname{dom} f$ and $E \subseteq \operatorname{dom} g$ and $f$ is measurable on $E$. Then $g$ is measurable on $E$.

(37) Let us consider non empty sets $X_{1}, X_{2}$, a subset $A$ of $X_{1} \times X_{2}$, a partial function $f$ from $X_{1} \times X_{2}$ to $\overline{\mathbb{R}}$, an element $x$ of $X_{1}$, an element $y$ of $X_{2}$, and a sequence $F$ of partial functions from $X_{1} \times X_{2}$ into $\overline{\mathbb{R}}$. Suppose $A \subseteq \operatorname{dom} f$ and for every natural number $n, \operatorname{dom}(F(n))=A$ and for every element $z$ of $X_{1} \times X_{2}$ such that $z \in A$ holds $F \# z$ is convergent and $\lim (F \# z)=f(z)$. Then

(i) there exists a sequence $F_{1}$ of partial functions from $X_{1}$ into $\overline{\mathbb{R}}$ with the same dom such that for every natural number $n, F_{1}(n)=$ ProjPMap2 $(F(n), y)$ and for every element $x$ of $X_{1}$ such that $x \in \operatorname{Ysection}(A, y)$ holds $F_{1} \# x$ is convergent and $(\operatorname{ProjPMap} 2(f, y))(x)=\lim \left(F_{1} \# x\right)$, and

(ii) there exists a sequence $F_{2}$ of partial functions from $X_{2}$ into $\overline{\mathbb{R}}$ with the same dom such that for every natural number $n, F_{2}(n)=$ ProjPMap1 $(F(n), x)$ and for every element $y$ of $X_{2}$ such that $y \in \mathrm{X} \operatorname{section}(A, x)$ holds $F_{2} \# y$ is convergent and $(\operatorname{ProjPMap} 1(f, x))(y)=\lim \left(F_{2} \# y\right)$.

Proof: Define $\mathcal{R}$ [element of $\mathbb{N}$, object $] \equiv \$_{2}=\operatorname{ProjPMap} 2\left(F\left(\$_{1}\right), y\right)$. For every element $n$ of $\mathbb{N}$, there exists an element $f$ of $X_{1} \rightarrow \overline{\mathbb{R}}$ such that 
$\mathcal{R}[n, f]$. There exists a sequence $F_{1}$ of partial functions from $X_{1}$ into $\overline{\mathbb{R}}$ with the same dom such that for every natural number $n, F_{1}(n)=$ ProjPMap2 $(F(n), y)$ and for every element $x$ of $X_{1}$ such that $x \in$ Ysection $(A, y)$ holds $F_{1} \# x$ is convergent and $(\operatorname{ProjPMap} 2(f, y))(x)=\lim \left(F_{1} \# x\right)$. Define $\mathcal{Q}$ [element of $\mathbb{N}$, object] $\equiv \$_{2}=\operatorname{ProjPMap} 1\left(F\left(\$_{1}\right), x\right)$. For every element $n$ of $\mathbb{N}$, there exists an element $f$ of $X_{2} \dot{\rightarrow} \overline{\mathbb{R}}$ such that $\mathcal{Q}[n, f]$. Consider $F_{2}$ being a sequence of $X_{2} \dot{\rightarrow} \overline{\mathbb{R}}$ such that for every element $n$ of $\mathbb{N}$, $\mathcal{Q}\left[n, F_{2}(n)\right]$. For every natural number $n, \operatorname{dom}\left(F_{2}(n)\right)=\operatorname{Xsection}(A, x)$. For every natural numbers $m, n, \operatorname{dom}\left(F_{2}(m)\right)=\operatorname{dom}\left(F_{2}(n)\right)$. For every natural number $n, F_{2}(n)=\operatorname{ProjPMap} 1(F(n), x)$. For every element $y$ of $X_{2}$ such that $y \in \mathrm{X} \operatorname{section}(A, x)$ holds $F_{2} \# y$ is convergent and $(\operatorname{ProjPMap} 1(f, x))(y)=\lim \left(F_{2} \# y\right)$.

(38) Let us consider non empty sets $X_{1}, X_{2}$, a $\sigma$-field $S_{1}$ of subsets of $X_{1}$, a $\sigma$-field $S_{2}$ of subsets of $X_{2}$, an element $E$ of $\sigma\left(\operatorname{MeasRect}\left(S_{1}, S_{2}\right)\right)$, a $\sigma$ measure $M_{2}$ on $S_{2}$, an element $A$ of $S_{1}$, an element $B$ of $S_{2}$, and an element $x$ of $X_{1}$. Then $M_{2}(B \cap$ MeasurableXsection $(E, x)) \cdot\left(\chi_{A, X_{1}}(x)\right)=$ $\int \operatorname{ProjPMap} 1\left(\chi_{A \times B, X_{1} \times X_{2}} \uparrow E, x\right) \mathrm{d} M_{2}$.

Proof: Set $C_{1}=\chi_{A \times B, X_{1} \times X_{2}}\left\lceil E\right.$. ProjPMap1 $\left(\chi_{A \times B, X_{1} \times X_{2}}, x\right)=$ $\operatorname{curry}\left(\chi_{A \times B, X_{1} \times X_{2}}, x\right)$. ProjPMap1 $\left(C_{1}, x\right)=$

$\operatorname{ProjPMap} 1\left(\chi_{A \times B, X_{1} \times X_{2}}, x\right)\left\lceil\mathrm{X} \operatorname{section}(E, x)\right.$. For every element $y$ of $X_{2}$, $\left(\operatorname{ProjPMap} 1\left(C_{1}, x\right)\right)(y)=$

$\left(\chi_{A, X_{1}} \uparrow\right.$ MeasurableYsection $\left.(E, y)\right)(x) \cdot\left(\chi_{B, X_{2}}(y)\right)$.

(39) Let us consider non empty sets $X_{1}, X_{2}$, a $\sigma$-field $S_{1}$ of subsets of $X_{1}$, a $\sigma$-field $S_{2}$ of subsets of $X_{2}$, an element $E$ of $\sigma\left(\operatorname{MeasRect}\left(S_{1}, S_{2}\right)\right)$, a $\sigma$ measure $M_{1}$ on $S_{1}$, an element $A$ of $S_{1}$, an element $B$ of $S_{2}$, and an element $y$ of $X_{2}$. Then $M_{1}(A \cap$ MeasurableYsection $(E, y)) \cdot\left(\chi_{B, X_{2}}(y)\right)=$ $\int \operatorname{ProjPMap} 2\left(\chi_{A \times B, X_{1} \times X_{2}} \uparrow E, y\right) \mathrm{d} M_{1}$.

Proof: Set $C_{1}=\chi_{A \times B, X_{1} \times X_{2}}\left\lceil E\right.$. ProjPMap2 $\left(\chi_{A \times B, X_{1} \times X_{2}}, y\right)=$ $\operatorname{curry}^{\prime}\left(\chi_{A \times B, X_{1} \times X_{2}}, y\right)$. ProjPMap2 $\left(C_{1}, y\right)=$

$\operatorname{ProjPMap} 2\left(\chi_{A \times B, X_{1} \times X_{2}}, y\right)\left\lceil\operatorname{Ysection}(E, y)\right.$. For every element $x$ of $X_{1}$, $\left(\operatorname{ProjPMap} 2\left(C_{1}, y\right)\right)(x)=$

$\left(\chi_{B, X_{2}} \uparrow \operatorname{MeasurableX} \operatorname{section}(E, x)\right)(y) \cdot\left(\chi_{A, X_{1}}(x)\right)$ by [4, (2)].

(40) Let us consider non empty sets $X_{1}, X_{2}$, an element $x$ of $X_{1}$, an element $y$ of $X_{2}$, a partial function $f$ from $X_{1} \times X_{2}$ to $\overline{\mathbb{R}}$, and an extended real $e$. Then

(i) $\langle x, y\rangle \in \operatorname{dom} f$ and $f(x, y)=e$ iff $y \in \operatorname{dom} \operatorname{ProjPMap} 1(f, x)$ and $(\operatorname{ProjPMap} 1(f, x))(y)=e$, and

(ii) $\langle x, y\rangle \in \operatorname{dom} f$ and $f(x, y)=e$ iff $x \in \operatorname{dom} \operatorname{ProjPMap} 2(f, y)$ and $(\operatorname{ProjPMap} 2(f, y))(x)=e$. 
The theorem is a consequence of (25) and (26).

(41) Let us consider non empty sets $X_{1}, X_{2}$, sets $A, Z$, a partial function $f$ from $X_{1} \times X_{2}$ to $Z$, and an element $x$ of $X_{1}$. Then $X \operatorname{section}\left(f^{-1}(A), x\right)=$ $(\operatorname{ProjPMap} 1(f, x))^{-1}(A)$.

(42) Let us consider non empty sets $X_{1}, X_{2}$, sets $A, Z$, a partial function $f$ from $X_{1} \times X_{2}$ to $Z$, and an element $y$ of $X_{2}$. Then $Y$ section $\left(f^{-1}(A), y\right)=$ (ProjPMap2 $(f, y))^{-1}(A)$.

(43) Let us consider non empty sets $X_{1}, X_{2}$, subsets $A, B$ of $X_{1} \times X_{2}$, and a set $p$. Then

(i) $\mathrm{Xsection}(A \backslash B, p)=\mathrm{X} \operatorname{section}(A, p) \backslash \mathrm{X} \operatorname{section}(B, p)$, and

(ii) $\operatorname{Ysection}(A \backslash B, p)=\mathrm{Ysection}(A, p) \backslash \mathrm{Y} \operatorname{section}(B, p)$.

(44) Let us consider non empty sets $X_{1}, X_{2}$, an element $x$ of $X_{1}$, an element $y$ of $X_{2}$, and partial functions $f_{1}, f_{2}$ from $X_{1} \times X_{2}$ to $\overline{\mathbb{R}}$. Then

(i) $\operatorname{ProjPMap} 1\left(f_{1}+f_{2}, x\right)=\operatorname{ProjPMap} 1\left(f_{1}, x\right)+\operatorname{ProjPMap} 1\left(f_{2}, x\right)$, and

(ii) $\operatorname{ProjPMap} 1\left(f_{1}-f_{2}, x\right)=\operatorname{ProjPMap} 1\left(f_{1}, x\right)-\operatorname{ProjPMap} 1\left(f_{2}, x\right)$, and

(iii) ProjPMap2 $\left(f_{1}+f_{2}, y\right)=\operatorname{ProjPMap} 2\left(f_{1}, y\right)+\operatorname{ProjPMap} 2\left(f_{2}, y\right)$, and

(iv) ProjPMap2 $\left(f_{1}-f_{2}, y\right)=\operatorname{ProjPMap} 2\left(f_{1}, y\right)-\operatorname{ProjPMap} 2\left(f_{2}, y\right)$.

The theorem is a consequence of (42), (41), (43), (26), and (40).

(45) Let us consider non empty sets $X_{1}, X_{2}$, a partial function $f$ from $X_{1} \times$ $X_{2}$ to $\overline{\mathbb{R}}$, and an element $x$ of $X_{1}$. Then

(i) ProjPMap1 $\left(\max _{+}(f), x\right)=\max _{+}(\operatorname{ProjPMap} 1(f, x))$, and

(ii) $\operatorname{ProjPMap1}\left(\max _{-}(f), x\right)=\max _{-}(\operatorname{ProjPMap}(f, x))$.

(46) Let us consider non empty sets $X_{1}, X_{2}$, a partial function $f$ from $X_{1} \times$ $X_{2}$ to $\overline{\mathbb{R}}$, and an element $y$ of $X_{2}$. Then

(i) ProjPMap2 $\left(\max _{+}(f), y\right)=\max _{+}(\operatorname{ProjPMap} 2(f, y))$, and

(ii) ProjPMap2 $\left(\max _{-}(f), y\right)=\max _{-}\left(\operatorname{ProjPMap}_{2}(f, y)\right)$.

(47) Let us consider non empty sets $X_{1}, X_{2}$, a $\sigma$-field $S_{1}$ of subsets of $X_{1}$, a $\sigma$ field $S_{2}$ of subsets of $X_{2}$, a partial function $f$ from $X_{1} \times X_{2}$ to $\overline{\mathbb{R}}$, an element $x$ of $X_{1}$, an element $y$ of $X_{2}$, and an element $E$ of $\sigma\left(\operatorname{MeasRect}\left(S_{1}, S_{2}\right)\right)$. Suppose $E \subseteq \operatorname{dom} f$ and $f$ is measurable on $E$. Then

(i) $\operatorname{ProjPMap} 1(f, x)$ is measurable on MeasurableXsection $(E, x)$, and

(ii) ProjPMap2 $(f, y)$ is measurable on MeasurableYsection $(E, y)$.

The theorem is a consequence of (45) and (46). 
Let $X_{1}, X_{2}, Y$ be non empty sets, $F$ be a sequence of partial functions from $X_{1} \times X_{2}$ into $Y$, and $x$ be an element of $X_{1}$. The functor $\operatorname{ProjPMap} 1(F, x)$ yielding a sequence of partial functions from $X_{2}$ into $Y$ is defined by

(Def. 5) for every natural number $n, i t(n)=\operatorname{ProjPMap} 1(F(n), x)$.

Let $y$ be an element of $X_{2}$. The functor ProjPMap2 $(F, y)$ yielding a sequence of partial functions from $X_{1}$ into $Y$ is defined by

(Def. 6) for every natural number $n$, it $(n)=\operatorname{ProjPMap} 2(F(n), y)$.

(48) Let us consider non empty sets $X_{1}, X_{2}$, a subset $E$ of $X_{1} \times X_{2}$, an element $x$ of $X_{1}$, and an element $y$ of $X_{2}$. Then

(i) $\operatorname{ProjPMap} 1\left(\chi_{E, X_{1} \times X_{2}}, x\right)=\chi_{\mathrm{Xsection}(E, x), X_{2}}$, and

(ii) $\operatorname{ProjPMap} 2\left(\chi_{E, X_{1} \times X_{2}}, y\right)=\chi_{\mathrm{Ysection}(E, y), X_{1}}$.

The theorem is a consequence of (25) and (27).

Let us consider a non empty set $X$, a $\sigma$-field $S$ of subsets of $X$, a $\sigma$-measure $M$ on $S$, an element $E$ of $S$, and an extended real $e$. Now we state the propositions:

(49) $\int \chi_{e, E, X} \mathrm{~d} M=e \cdot M(E)$. The theorem is a consequence of (2), (12), and (1).

(50) $\int \chi_{e, E, X}\lceil E \mathrm{~d} M=e \cdot M(E)$. The theorem is a consequence of (15), (2), (13), (49), (16), (1), and (12).

(51) Let us consider a non empty set $X$, a $\sigma$-field $S$ of subsets of $X$, a $\sigma$ measure $M$ on $S$, elements $E_{1}, E_{2}$ of $S$, and an extended real $e$. Then $\int \chi_{e, E_{1}, X}\left\lceil E_{2} \mathrm{~d} M=e \cdot M\left(E_{1} \cap E_{2}\right)\right.$. The theorem is a consequence of (14), (17), (13), (16), (15), and (50).

(52) Let us consider non empty sets $X_{1}, X_{2}$, a $\sigma$-field $S_{1}$ of subsets of $X_{1}$, a $\sigma$-field $S_{2}$ of subsets of $X_{2}$, a $\sigma$-measure $M_{2}$ on $S_{2}$, an element $x$ of $X_{1}$, and an element $E$ of $\sigma\left(\operatorname{MeasRect}\left(S_{1}, S_{2}\right)\right)$. Suppose $M_{2}$ is $\sigma$-finite. Then

(i) $\left(\operatorname{Yvol}\left(E, M_{2}\right)\right)(x)=\int \operatorname{ProjPMap1}\left(\chi_{E, X_{1} \times X_{2}}, x\right) \mathrm{d} M_{2}$, and

(ii) $\left(\operatorname{Yvol}\left(E, M_{2}\right)\right)(x)=\int^{+} \operatorname{ProjPMap1}\left(\chi_{E, X_{1} \times X_{2}}, x\right) \mathrm{d} M_{2}$, and

(iii) $\left(\operatorname{Yvol}\left(E, M_{2}\right)\right)(x)=\int^{\prime} \operatorname{ProjPMap1}\left(\chi_{E, X_{1} \times X_{2}}, x\right) \mathrm{d} M_{2}$.

The theorem is a consequence of (48), (12), and (27).

(53) Let us consider non empty sets $X_{1}, X_{2}$, a $\sigma$-field $S_{1}$ of subsets of $X_{1}$, a $\sigma$-field $S_{2}$ of subsets of $X_{2}$, a $\sigma$-measure $M_{1}$ on $S_{1}$, an element $y$ of $X_{2}$, and an element $E$ of $\sigma\left(\operatorname{MeasRect}\left(S_{1}, S_{2}\right)\right)$. Suppose $M_{1}$ is $\sigma$-finite. Then

(i) $\left(\mathrm{X}_{\operatorname{vol}}\left(E, M_{1}\right)\right)(y)=\int \operatorname{ProjPMap} 2\left(\chi_{E, X_{1} \times X_{2}}, y\right) \mathrm{d} M_{1}$, and

(ii) $\left(\operatorname{Xvol}\left(E, M_{1}\right)\right)(y)=\int^{+} \operatorname{ProjPMap} 2\left(\chi_{E, X_{1} \times X_{2}}, y\right) \mathrm{d} M_{1}$, and

(iii) $\left(\mathrm{Xvol}\left(E, M_{1}\right)\right)(y)=\int^{\prime} \operatorname{ProjPMap} 2\left(\chi_{E, X_{1} \times X_{2}}, y\right) \mathrm{d} M_{1}$.

The theorem is a consequence of (48), (12), and (27). 
(54) Let us consider a non empty set $X$, a $\sigma$-field $S$ of subsets of $X$, a $\sigma$ measure $M$ on $S$, an element $E$ of $S$, and a real number $r$. Then $\int r$. $\chi_{E, X} \mathrm{~d} M=r \cdot \int \chi_{E, X} \mathrm{~d} M$. The theorem is a consequence of (12).

(55) Let us consider non empty sets $X_{1}, X_{2}$, a $\sigma$-field $S_{1}$ of subsets of $X_{1}$, a $\sigma$-field $S_{2}$ of subsets of $X_{2}$, a $\sigma$-measure $M_{1}$ on $S_{1}$, an element $y$ of $X_{2}$, an element $E$ of $\sigma\left(\operatorname{MeasRect}\left(S_{1}, S_{2}\right)\right)$, and a real number $r$. Suppose $M_{1}$ is $\sigma$-finite. Then

(i) $\left(r \cdot \mathrm{Xvol}\left(E, M_{1}\right)\right)(y)=\int \operatorname{ProjPMap} 2\left(\chi_{r, E, X_{1} \times X_{2}}, y\right) \mathrm{d} M_{1}$, and

(ii) if $r \geqslant 0$, then $\left(r \cdot \mathrm{Xvol}\left(E, M_{1}\right)\right)(y)=\int^{+} \operatorname{ProjPMap} 2\left(\chi_{r, E, X_{1} \times X_{2}}, y\right) \mathrm{d} M_{1}$.

Proof: Set $p_{2}=\operatorname{ProjPMap} 2\left(\chi_{E, X_{1} \times X_{2}}, y\right) \cdot \chi_{r, E, X_{1} \times X_{2}}=r \cdot \chi_{E, X_{1} \times X_{2}}$. ProjPMap2 $\left(\chi_{r, E, X_{1} \times X_{2}}, y\right)=r \cdot p_{2} \cdot p_{2}$ is non-negative. $\chi_{E, X_{1} \times X_{2}}$ is simple function in $\sigma\left(\operatorname{MeasRect}\left(S_{1}, S_{2}\right)\right)$. $\int \operatorname{ProjPMap2}\left(\chi_{r, E, X_{1} \times X_{2}}, y\right) \mathrm{d} M_{1}=r$. $\left(\int^{\prime} p_{2} \mathrm{~d} M_{1}\right)$. If $r \geqslant 0$, then $\left(r \cdot \operatorname{Xvol}\left(E, M_{1}\right)\right)(y)=$ $\int^{+} \operatorname{ProjPMap} 2\left(\chi_{r, E, X_{1} \times X_{2}}, y\right) \mathrm{d} M_{1}$.

(56) Let us consider non empty sets $X_{1}, X_{2}$, a $\sigma$-field $S_{1}$ of subsets of $X_{1}$, a $\sigma$-field $S_{2}$ of subsets of $X_{2}$, a $\sigma$-measure $M_{2}$ on $S_{2}$, an element $x$ of $X_{1}$, an element $E$ of $\sigma\left(\operatorname{MeasRect}\left(S_{1}, S_{2}\right)\right)$, and a real number $r$. Suppose $M_{2}$ is $\sigma$-finite. Then

(i) $\left(r \cdot \operatorname{Yvol}\left(E, M_{2}\right)\right)(x)=\int \operatorname{ProjPMap1}\left(\chi_{r, E, X_{1} \times X_{2}}, x\right) \mathrm{d} M_{2}$, and

(ii) if $r \geqslant 0$, then $\left(r \cdot \operatorname{Yvol}\left(E, M_{2}\right)\right)(x)=\int^{+} \operatorname{ProjPMap} 1\left(\chi_{r, E, X_{1} \times X_{2}}, x\right) \mathrm{d} M_{2}$.

Proof: Set $p_{2}=\operatorname{ProjPMap1}\left(\chi_{E, X_{1} \times X_{2}}, x\right) . \chi_{r, E, X_{1} \times X_{2}}=r \cdot \chi_{E, X_{1} \times X_{2}}$. $\operatorname{ProjPMap} 1\left(\chi_{r, E, X_{1} \times X_{2}}, x\right)=r \cdot p_{2} \cdot p_{2}$ is non-negative. $\chi_{E, X_{1} \times X_{2}}$ is simple function in $\sigma\left(\operatorname{MeasRect}\left(S_{1}, S_{2}\right)\right)$. $\int \operatorname{ProjPMap1}\left(\chi_{r, E, X_{1} \times X_{2}}, x\right) \mathrm{d} M_{2}=r$. $\left(\int^{\prime} p_{2} \mathrm{~d} M_{2}\right)$. If $r \geqslant 0$, then $\left(r \cdot \operatorname{Yvol}\left(E, M_{2}\right)\right)(x)=$ $\int^{+} \operatorname{ProjPMap} 1\left(\chi_{r, E, X_{1} \times X_{2}}, x\right) \mathrm{d} M_{2}$.

(57) Let us consider a non empty set $X$, a $\sigma$-field $S$ of subsets of $X$, a $\sigma$ measure $M$ on $S$, and a partial function $f$ from $X$ to $\overline{\mathbb{R}}$. Suppose $\operatorname{dom} f \in S$ and for every element $x$ of $X$ such that $x \in \operatorname{dom} f$ holds $0=f(x)$. Then

(i) for every element $E$ of $S$ such that $E \subseteq \operatorname{dom} f$ holds $f$ is measurable on $E$, and

(ii) $\int f \mathrm{~d} M=0$.

The theorem is a consequence of (15) and (50).

(58) Let us consider non empty sets $X_{1}, X_{2}, Y$, a sequence $F$ of partial functions from $X_{1} \times X_{2}$ into $Y$, an element $x$ of $X_{1}$, and an element $y$ of $X_{2}$. Suppose $F$ has the same dom. Then

(i) $\operatorname{ProjPMap1}(F, x)$ has the same dom, and

(ii) ProjPMap2 $(F, y)$ has the same dom. 


\section{Fubini's Theorem for Non-negative or Non-Positive Functions}

Let $X_{1}, X_{2}$ be non empty sets, $S_{1}$ be a $\sigma$-field of subsets of $X_{1}, M_{1}$ be a $\sigma$ measure on $S_{1}$, and $f$ be a partial function from $X_{1} \times X_{2}$ to $\overline{\mathbb{R}}$. The functor Integral1 $\left(M_{1}, f\right)$ yielding a function from $X_{2}$ into $\overline{\mathbb{R}}$ is defined by

(Def. 7) for every element $y$ of $X_{2}, i t(y)=\int \operatorname{ProjPMap} 2(f, y) \mathrm{d} M_{1}$.

Let $S_{2}$ be a $\sigma$-field of subsets of $X_{2}$ and $M_{2}$ be a $\sigma$-measure on $S_{2}$. The functor Integral2 $\left(M_{2}, f\right)$ yielding a function from $X_{1}$ into $\overline{\mathbb{R}}$ is defined by

(Def. 8) for every element $x$ of $X_{1}$, it $(x)=\int \operatorname{ProjPMap} 1(f, x) \mathrm{d} M_{2}$.

Now we state the propositions:

(59) Let us consider non empty sets $X_{1}, X_{2}$, a $\sigma$-field $S_{1}$ of subsets of $X_{1}$, a $\sigma$-field $S_{2}$ of subsets of $X_{2}$, a $\sigma$-measure $M_{1}$ on $S_{1}$, a partial function $f$ from $X_{1} \times X_{2}$ to $\overline{\mathbb{R}}$, an element $E$ of $\sigma$ (MeasRect $\left(S_{1}, S_{2}\right)$ ), and an element $V$ of $S_{2}$. Suppose $M_{1}$ is $\sigma$-finite and $f$ is non-negative or non-positive and $E=\operatorname{dom} f$ and $f$ is measurable on $E$. Then $\operatorname{Integral1}\left(M_{1}, f\right)$ is measurable on $V$.

(60) Let us consider non empty sets $X_{1}, X_{2}$, a $\sigma$-field $S_{1}$ of subsets of $X_{1}$, a $\sigma$-field $S_{2}$ of subsets of $X_{2}$, a $\sigma$-measure $M_{2}$ on $S_{2}$, a partial function $f$ from $X_{1} \times X_{2}$ to $\overline{\mathbb{R}}$, an element $E$ of $\sigma$ (MeasRect $\left.\left(S_{1}, S_{2}\right)\right)$, and an element $U$ of $S_{1}$. Suppose $M_{2}$ is $\sigma$-finite and $f$ is non-negative or non-positive and $E=\operatorname{dom} f$ and $f$ is measurable on $E$. Then Integral2 $\left(M_{2}, f\right)$ is measurable on $U$.

(61) Let us consider non empty sets $X_{1}, X_{2}$, a $\sigma$-field $S_{1}$ of subsets of $X_{1}$, a $\sigma$-field $S_{2}$ of subsets of $X_{2}$, a $\sigma$-measure $M_{1}$ on $S_{1}$, an element $y$ of $X_{2}$, and an element $E$ of $\sigma\left(\operatorname{MeasRect}\left(S_{1}, S_{2}\right)\right)$. Suppose $M_{1}$ is $\sigma$-finite. Then $\left(\mathrm{X} \operatorname{vol}\left(E, M_{1}\right)\right)(y)=\int \chi_{\text {MeasurableYsection }(E, y), X_{1}} \mathrm{~d} M_{1}$.

(62) Let us consider non empty sets $X_{1}, X_{2}$, a $\sigma$-field $S_{1}$ of subsets of $X_{1}$, a $\sigma$-field $S_{2}$ of subsets of $X_{2}$, a $\sigma$-measure $M_{2}$ on $S_{2}$, an element $x$ of $X_{1}$, and an element $E$ of $\sigma\left(\operatorname{MeasRect}\left(S_{1}, S_{2}\right)\right)$. Suppose $M_{2}$ is $\sigma$-finite. Then $\left(\operatorname{Yvol}\left(E, M_{2}\right)\right)(x)=\int \chi_{\text {MeasurableXsection }(E, x), X_{2}} \mathrm{~d} M_{2}$.

(63) Let us consider non empty sets $X_{1}, X_{2}$, a $\sigma$-field $S_{1}$ of subsets of $X_{1}$, a $\sigma$ field $S_{2}$ of subsets of $X_{2}$, an element $E$ of $\sigma\left(\right.$ MeasRect $\left(S_{1}, S_{2}\right)$ ), an element $x$ of $X_{1}$, and an element $y$ of $X_{2}$. Then

(i) ProjPMap1 $\left(\chi_{E, X_{1} \times X_{2}}, x\right)=\chi_{\text {MeasurableXsection }(E, x), X_{2}}$, and

(ii) ProjPMap2 $\left(\chi_{E, X_{1} \times X_{2}}, y\right)=\chi_{\text {MeasurableYsection }(E, y), X_{1}}$.

(64) Let us consider non empty sets $X_{1}, X_{2}$, a $\sigma$-field $S_{1}$ of subsets of $X_{1}$, a $\sigma$-field $S_{2}$ of subsets of $X_{2}$, a $\sigma$-measure $M_{1}$ on $S_{1}$, and an element 
$E$ of $\sigma\left(\operatorname{MeasRect}\left(S_{1}, S_{2}\right)\right)$. Suppose $M_{1}$ is $\sigma$-finite. Then $\operatorname{Xvol}\left(E, M_{1}\right)=$ Integral1 $\left(M_{1}, \chi_{E, X_{1} \times X_{2}}\right)$. The theorem is a consequence of (61) and (63).

(65) Let us consider non empty sets $X_{1}, X_{2}$, a $\sigma$-field $S_{1}$ of subsets of $X_{1}$, a $\sigma$-field $S_{2}$ of subsets of $X_{2}$, a $\sigma$-measure $M_{2}$ on $S_{2}$, and an element $E$ of $\sigma\left(\operatorname{MeasRect}\left(S_{1}, S_{2}\right)\right)$. Suppose $M_{2}$ is $\sigma$-finite. Then $\operatorname{Yvol}\left(E, M_{2}\right)=$ Integral2 $\left(M_{2}, \chi_{E, X_{1} \times X_{2}}\right)$. The theorem is a consequence of (62) and (63).

Let $X_{1}, X_{2}$ be non empty sets, $S_{1}$ be a $\sigma$-field of subsets of $X_{1}, S_{2}$ be a $\sigma$ field of subsets of $X_{2}, M_{1}$ be a $\sigma$-measure on $S_{1}$, and $M_{2}$ be a $\sigma$-measure on $S_{2}$. The functor $\operatorname{ProdMeas}\left(M_{1}, M_{2}\right)$ yielding a $\sigma$-measure on $\sigma\left(\operatorname{MeasRect}\left(S_{1}, S_{2}\right)\right)$ is defined by the term

(Def. 9) $\operatorname{Prod} \sigma-\operatorname{Meas}\left(M_{1}, M_{2}\right)$.

Let us consider non empty sets $X_{1}, X_{2}$, a $\sigma$-field $S_{1}$ of subsets of $X_{1}$, a $\sigma$-field $S_{2}$ of subsets of $X_{2}$, a $\sigma$-measure $M_{1}$ on $S_{1}$, a $\sigma$-measure $M_{2}$ on $S_{2}$, a partial function $f$ from $X_{1} \times X_{2}$ to $\overline{\mathbb{R}}$, and elements $E_{1}, E_{2}$ of $\sigma\left(\operatorname{MeasRect}\left(S_{1}, S_{2}\right)\right)$. Now we state the propositions:

(66) Suppose $E_{1}=\operatorname{dom} f$ and $f$ is non-negative and $f$ is measurable on $E_{1}$. Then

(i) Integral1 $\left(M_{1}, f\right)$ is non-negative, and

(ii) Integral1 $\left(M_{1}, f\left\lceil E_{2}\right)\right.$ is non-negative, and

(iii) Integral2 $\left(M_{2}, f\right)$ is non-negative, and

(iv) Integral2 $\left(M_{2}, f \nmid E_{2}\right)$ is non-negative.

The theorem is a consequence of (47) and (32).

(67) Suppose $E_{1}=\operatorname{dom} f$ and $f$ is non-positive and $f$ is measurable on $E_{1}$. Then

(i) Integral1 $\left(M_{1}, f\right)$ is non-positive, and

(ii) Integral1 $\left(M_{1}, f\left\lceil E_{2}\right)\right.$ is non-positive, and

(iii) Integral2 $\left(M_{2}, f\right)$ is non-positive, and

(iv) Integral2 $\left(M_{2}, f\left\lceil E_{2}\right)\right.$ is non-positive.

The theorem is a consequence of (47) and (33).

(68) Let us consider non empty sets $X_{1}, X_{2}$, a $\sigma$-field $S_{1}$ of subsets of $X_{1}$, a $\sigma$ field $S_{2}$ of subsets of $X_{2}$, a $\sigma$-measure $M_{1}$ on $S_{1}$, a partial function $f$ from $X_{1} \times X_{2}$ to $\overline{\mathbb{R}}$, elements $E_{1}, E_{2}$ of $\sigma\left(\operatorname{MeasRect}\left(S_{1}, S_{2}\right)\right)$, and an element $V$ of $S_{2}$. Suppose $M_{1}$ is $\sigma$-finite and $f$ is non-negative or non-positive and $E_{1}=\operatorname{dom} f$ and $f$ is measurable on $E_{1}$. Then $\operatorname{Integral1}\left(M_{1}, f\left\lceil E_{2}\right)\right.$ is measurable on $V$. The theorem is a consequence of (59). 
(69) Let us consider non empty sets $X_{1}, X_{2}$, a $\sigma$-field $S_{1}$ of subsets of $X_{1}$, a $\sigma$ field $S_{2}$ of subsets of $X_{2}$, a $\sigma$-measure $M_{2}$ on $S_{2}$, a partial function $f$ from $X_{1} \times X_{2}$ to $\overline{\mathbb{R}}$, elements $E_{1}, E_{2}$ of $\sigma\left(\right.$ MeasRect $\left.\left(S_{1}, S_{2}\right)\right)$, and an element $U$ of $S_{1}$. Suppose $M_{2}$ is $\sigma$-finite and $f$ is non-negative or non-positive and $E_{1}=\operatorname{dom} f$ and $f$ is measurable on $E_{1}$. Then Integral2 $\left(M_{2}, f\left\lceil E_{2}\right)\right.$ is measurable on $U$. The theorem is a consequence of (60).

(70) Let us consider non empty sets $X_{1}, X_{2}$, a $\sigma$-field $S_{1}$ of subsets of $X_{1}$, a $\sigma$-field $S_{2}$ of subsets of $X_{2}$, a $\sigma$-measure $M_{1}$ on $S_{1}$, a partial function $f$ from $X_{1} \times X_{2}$ to $\overline{\mathbb{R}}$, an element $E$ of $\sigma\left(\operatorname{MeasRect}\left(S_{1}, S_{2}\right)\right)$, and an element $y$ of $X_{2}$. Suppose $E=\operatorname{dom} f$ and $f$ is non-negative or nonpositive and $f$ is measurable on $E$ and for every element $x$ of $X_{1}$ such that $x \in \operatorname{dom} \operatorname{ProjPMap} 2(f, y)$ holds $(\operatorname{ProjPMap} 2(f, y))(x)=0$. Then (Integral1 $\left.\left(M_{1}, f\right)\right)(y)=0$. The theorem is a consequence of $(47),(32)$, and (33).

(71) Let us consider non empty sets $X_{1}, X_{2}$, a $\sigma$-field $S_{1}$ of subsets of $X_{1}$, a $\sigma$-field $S_{2}$ of subsets of $X_{2}$, a $\sigma$-measure $M_{2}$ on $S_{2}$, a partial function $f$ from $X_{1} \times X_{2}$ to $\overline{\mathbb{R}}$, an element $E$ of $\sigma\left(\operatorname{MeasRect}\left(S_{1}, S_{2}\right)\right)$, and an element $x$ of $X_{1}$. Suppose $E=\operatorname{dom} f$ and $f$ is non-negative or nonpositive and $f$ is measurable on $E$ and for every element $y$ of $X_{2}$ such that $y \in \operatorname{dom} \operatorname{ProjPMap} 1(f, x)$ holds $(\operatorname{ProjPMap} 1(f, x))(y)=0$. Then (Integral2 $\left.\left(M_{2}, f\right)\right)(x)=0$. The theorem is a consequence of (47), (32), and (33).

(72) Let us consider non empty sets $X_{1}, X_{2}$, a $\sigma$-field $S_{1}$ of subsets of $X_{1}$, a $\sigma$ field $S_{2}$ of subsets of $X_{2}$, a $\sigma$-measure $M_{1}$ on $S_{1}$, a $\sigma$-measure $M_{2}$ on $S_{2}$, elements $E, E_{1}, E_{2}$ of $\sigma$ (MeasRect $\left(S_{1}, S_{2}\right)$ ), and a partial function $f$ from $X_{1} \times X_{2}$ to $\overline{\mathbb{R}}$. Suppose $E=\operatorname{dom} f$ and $f$ is non-negative or non-positive and $f$ is measurable on $E$ and $E_{1}$ misses $E_{2}$. Then

(i) $\operatorname{Integral1}\left(M_{1}, f \uparrow\left(E_{1} \cup E_{2}\right)\right)=$ $\operatorname{Integral1}\left(M_{1}, f\left\lceil E_{1}\right)+\operatorname{Integral1}\left(M_{1}, f\left\lceil E_{2}\right)\right.\right.$, and

(ii) Integral2 $\left(M_{2}, f \uparrow\left(E_{1} \cup E_{2}\right)\right)=$ Integral2 $\left(M_{2}, f\left\lceil E_{1}\right)+\operatorname{Integral} 2\left(M_{2}, f\left\lceil E_{2}\right)\right.\right.$.

(73) Let us consider non empty sets $X_{1}, X_{2}$, a $\sigma$-field $S_{1}$ of subsets of $X_{1}$, a $\sigma$-field $S_{2}$ of subsets of $X_{2}$, a $\sigma$-measure $M_{1}$ on $S_{1}$, a $\sigma$-measure $M_{2}$ on $S_{2}$, a partial function $f$ from $X_{1} \times X_{2}$ to $\overline{\mathbb{R}}$, and an element $E$ of $\sigma\left(\right.$ MeasRect $\left.\left(S_{1}, S_{2}\right)\right)$. Suppose $E=\operatorname{dom} f$ and $f$ is measurable on $E$. Then

(i) Integral1 $\left(M_{1},-f\right)=-\operatorname{Integral1}\left(M_{1}, f\right)$, and

(ii) Integral2 $\left(M_{2},-f\right)=-\operatorname{Integral} 2\left(M_{2}, f\right)$. 
The theorem is a consequence of (29) and (47).

Let us consider non empty sets $X_{1}, X_{2}$, a $\sigma$-field $S_{1}$ of subsets of $X_{1}$, a $\sigma$ field $S_{2}$ of subsets of $X_{2}$, a $\sigma$-measure $M_{1}$ on $S_{1}$, a $\sigma$-measure $M_{2}$ on $S_{2}$, partial functions $f, g$ from $X_{1} \times X_{2}$ to $\overline{\mathbb{R}}$, and elements $E_{1}, E_{2}$ of $\sigma\left(\operatorname{MeasRect}\left(S_{1}, S_{2}\right)\right)$. Now we state the propositions:

(74) Suppose $E_{1}=\operatorname{dom} f$ and $f$ is non-negative and $f$ is measurable on $E_{1}$ and $E_{2}=\operatorname{dom} g$ and $g$ is non-negative and $g$ is measurable on $E_{2}$. Then

(i) $\operatorname{Integral1}\left(M_{1}, f+g\right)=$ $\operatorname{Integral1}\left(M_{1}, f\lceil\operatorname{dom}(f+g))+\operatorname{Integral1}\left(M_{1}, g\lceil\operatorname{dom}(f+g))\right.\right.$, and

(ii) Integral2 $\left(M_{2}, f+g\right)=$ $\operatorname{Integral2}\left(M_{2}, f\lceil\operatorname{dom}(f+g))+\operatorname{Integral} 2\left(M_{2}, g\lceil\operatorname{dom}(f+g))\right.\right.$.

Proof: Set $f_{1}=f \uparrow(A \cap B)$. Set $g_{1}=g \uparrow(A \cap B)$. Integral1 $\left(M_{1}, f_{1}\right)$ is nonnegative and Integral1 $\left(M_{1}, g_{1}\right)$ is non-negative and $\operatorname{Integral} 2\left(M_{2}, f_{1}\right)$ is non-negative and Integral2 $\left(M_{2}, g_{1}\right)$ is non-negative. For every element $y$ of $X_{2},\left(\operatorname{Integral1}\left(M_{1}, f_{1}\right)+\operatorname{Integral1}\left(M_{1}, g_{1}\right)\right)(y)=\left(\operatorname{Integral1}\left(M_{1}, f+g\right)\right)(y)$. For every element $x$ of $X_{1}$, (Integral2 $\left.\left(M_{2}, f_{1}\right)+\operatorname{Integral} 2\left(M_{2}, g_{1}\right)\right)(x)=$ (Integral2 $\left.\left(M_{2}, f+g\right)\right)(x)$.

(75) Suppose $E_{1}=\operatorname{dom} f$ and $f$ is non-positive and $f$ is measurable on $E_{1}$ and $E_{2}=\operatorname{dom} g$ and $g$ is non-positive and $g$ is measurable on $E_{2}$. Then

(i) $\operatorname{Integral1}\left(M_{1}, f+g\right)=$ $\operatorname{Integral1}\left(M_{1}, f\lceil\operatorname{dom}(f+g))+\operatorname{Integral1}\left(M_{1}, g\lceil\operatorname{dom}(f+g))\right.\right.$, and

(ii) Integral2 $\left(M_{2}, f+g\right)=$ Integral2 $\left(M_{2}, f\lceil\operatorname{dom}(f+g))+\operatorname{Integral} 2\left(M_{2}, g\lceil\operatorname{dom}(f+g))\right.\right.$.

The theorem is a consequence of (73) and (74).

(76) Suppose $E_{1}=\operatorname{dom} f$ and $f$ is non-negative and $f$ is measurable on $E_{1}$ and $E_{2}=\operatorname{dom} g$ and $g$ is non-positive and $g$ is measurable on $E_{2}$. Then

(i) $\operatorname{Integral1}\left(M_{1}, f-g\right)=$ $\operatorname{Integral1}\left(M_{1}, f\lceil\operatorname{dom}(f-g))-\operatorname{Integral1}\left(M_{1}, g\lceil\operatorname{dom}(f-g))\right.\right.$, and

(ii) Integral1 $\left(M_{1}, g-f\right)=$ $\operatorname{Integral1}\left(M_{1}, g\lceil\operatorname{dom}(g-f))-\operatorname{Integral1}\left(M_{1}, f\lceil\operatorname{dom}(g-f))\right.\right.$, and

(iii) Integral $2\left(M_{2}, f-g\right)=$ Integral2 $\left(M_{2}, f\lceil\operatorname{dom}(f-g))\right.$ - Integral2 $\left(M_{2}, g\lceil\operatorname{dom}(f-g))\right.$, and

(iv) Integral $2\left(M_{2}, g-f\right)=$ Integral2 $\left(M_{2}, g\lceil\operatorname{dom}(g-f))\right.$ - Integral2 $\left(M_{2}, f\lceil\operatorname{dom}(g-f))\right.$.

The theorem is a consequence of (74) and (73). 
(77) Let us consider non empty sets $X_{1}, X_{2}$, a $\sigma$-field $S_{1}$ of subsets of $X_{1}$, a $\sigma$-field $S_{2}$ of subsets of $X_{2}$, a $\sigma$-measure $M_{1}$ on $S_{1}$, a $\sigma$-measure $M_{2}$ on $S_{2}$, and an element $E$ of $\sigma\left(\operatorname{MeasRect}\left(S_{1}, S_{2}\right)\right)$. Suppose $M_{1}$ is $\sigma$-finite and $M_{2}$ is $\sigma$-finite. Then

(i) $\int \operatorname{Yvol}\left(E, M_{2}\right) \mathrm{d} M_{1}=\int \chi_{E, X_{1} \times X_{2}} \mathrm{~d} \operatorname{ProdMeas}\left(M_{1}, M_{2}\right)$, and

(ii) $\int \mathrm{X} \operatorname{vol}\left(E, M_{1}\right) \mathrm{d} M_{2}=\int \chi_{E, X_{1} \times X_{2}} \mathrm{~d} \operatorname{ProdMeas}\left(M_{1}, M_{2}\right)$.

(78) Let us consider non empty sets $X_{1}, X_{2}$, a $\sigma$-field $S_{1}$ of subsets of $X_{1}$, a $\sigma$-field $S_{2}$ of subsets of $X_{2}$, a $\sigma$-measure $M_{1}$ on $S_{1}$, a $\sigma$-measure $M_{2}$ on $S_{2}$, an element $E$ of $\sigma\left(\right.$ MeasRect $\left(S_{1}, S_{2}\right)$ ), a partial function $f$ from $X_{1} \times$ $X_{2}$ to $\overline{\mathbb{R}}$, and a real number $r$. Suppose $E=\operatorname{dom} f$ and $f$ is non-negative or non-positive and $f$ is measurable on $E$. Then

(i) Integral1 $\left(M_{1}, r \cdot f\right)=r \cdot \operatorname{Integral1}\left(M_{1}, f\right)$, and

(ii) Integral2 $\left(M_{2}, r \cdot f\right)=r \cdot \operatorname{Integral} 2\left(M_{2}, f\right)$.

The theorem is a consequence of (32), (33), (29), and (47).

Let us consider non empty sets $X_{1}, X_{2}$, a $\sigma$-field $S_{1}$ of subsets of $X_{1}$, a $\sigma$ field $S_{2}$ of subsets of $X_{2}$, a $\sigma$-measure $M_{1}$ on $S_{1}$, a $\sigma$-measure $M_{2}$ on $S_{2}$, and an element $E$ of $\sigma\left(\right.$ MeasRect $\left.\left(S_{1}, S_{2}\right)\right)$. Now we state the propositions:

(i) Integral1 $\left(M_{1}, \chi_{E, X_{1} \times X_{2}}\lceil E)=\operatorname{Integral1}\left(M_{1}, \chi_{E, X_{1} \times X_{2}}\right)\right.$, and

(ii) Integral2 $\left(M_{2}, \chi_{E, X_{1} \times X_{2}}\lceil E)=\operatorname{Integral} 2\left(M_{2}, \chi_{E, X_{1} \times X_{2}}\right)\right.$.

The theorem is a consequence of (34) and (48).

(80) (i) Integral1 $\left(M_{1}, \bar{\chi}_{E, X_{1} \times X_{2}}\lceil E)=\operatorname{Integral1}\left(M_{1}, \bar{\chi}_{E, X_{1} \times X_{2}}\right)\right.$, and

(ii) Integral2 $\left(M_{2}, \bar{\chi}_{E, X_{1} \times X_{2}}\lceil E)=\operatorname{Integral} 2\left(M_{2}, \bar{\chi}_{E, X_{1} \times X_{2}}\right)\right.$.

The theorem is a consequence of (34), (35), (2), (50), and (49).

(81) Let us consider non empty sets $X_{1}, X_{2}$, a $\sigma$-field $S_{1}$ of subsets of $X_{1}$, a $\sigma$-field $S_{2}$ of subsets of $X_{2}$, a $\sigma$-measure $M_{1}$ on $S_{1}$, a $\sigma$-measure $M_{2}$ on $S_{2}$, an element $E$ of $\sigma\left(\right.$ MeasRect $\left(S_{1}, S_{2}\right)$ ), and an extended real $e$. Then

(i) $\operatorname{Integral1}\left(M_{1}, \chi_{e, E, X_{1} \times X_{2}} \uparrow E\right)=\operatorname{Integral1}\left(M_{1}, \chi_{e, E, X_{1} \times X_{2}}\right)$, and

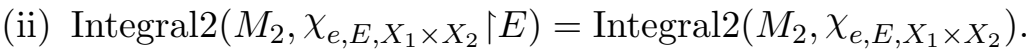

The theorem is a consequence of (1), (78), (79), (2), and (80).

(82) Let us consider non empty sets $X_{1}, X_{2}$, a $\sigma$-field $S_{1}$ of subsets of $X_{1}$, a $\sigma$-field $S_{2}$ of subsets of $X_{2}$, a $\sigma$-measure $M_{1}$ on $S_{1}$, a $\sigma$-measure $M_{2}$ on $S_{2}$, and an element $E$ of $\sigma\left(\operatorname{MeasRect}\left(S_{1}, S_{2}\right)\right)$. Suppose $M_{1}$ is $\sigma$-finite and $M_{2}$ is $\sigma$-finite. Then

(i) $\int \chi_{E, X_{1} \times X_{2}} \mathrm{~d} \operatorname{ProdMeas}\left(M_{1}, M_{2}\right)=\int \operatorname{Integral1}\left(M_{1}, \chi_{E, X_{1} \times X_{2}}\right) \mathrm{d} M_{2}$, and 
(ii) $\int \chi_{E, X_{1} \times X_{2}}\left\lceil E \mathrm{~d} \operatorname{ProdMeas}\left(M_{1}, M_{2}\right)=\right.$ $\int \operatorname{Integral1}\left(M_{1}, \chi_{E, X_{1} \times X_{2}}\lceil E) \mathrm{d} M_{2}\right.$, and

(iii) $\int \chi_{E, X_{1} \times X_{2}} \mathrm{~d} \operatorname{ProdMeas}\left(M_{1}, M_{2}\right)=\int \operatorname{Integral} 2\left(M_{2}, \chi_{E, X_{1} \times X_{2}}\right) \mathrm{d} M_{1}$, and

(iv) $\int \chi_{E, X_{1} \times X_{2}} \uparrow E \mathrm{~d} \operatorname{ProdMeas}\left(M_{1}, M_{2}\right)=$ $\int \operatorname{Integral} 2\left(M_{2}, \chi_{E, X_{1} \times X_{2}}\lceil E) \mathrm{d} M_{1}\right.$.

The theorem is a consequence of (64), (77), (79), and (65).

(83) Let us consider non empty sets $X_{1}, X_{2}$, a $\sigma$-field $S_{1}$ of subsets of $X_{1}$, a $\sigma$-field $S_{2}$ of subsets of $X_{2}$, a $\sigma$-measure $M_{1}$ on $S_{1}$, a $\sigma$-measure $M_{2}$ on $S_{2}$, an element $E$ of $\sigma$ (MeasRect $\left.\left(S_{1}, S_{2}\right)\right)$, and a real number $r$. Suppose $M_{1}$ is $\sigma$-finite and $M_{2}$ is $\sigma$-finite. Then

(i) $\int \chi_{r, E, X_{1} \times X_{2}} \mathrm{~d} \operatorname{ProdMeas}\left(M_{1}, M_{2}\right)=\int \operatorname{Integral1}\left(M_{1}, \chi_{r, E, X_{1} \times X_{2}}\right) \mathrm{d} M_{2}$, and

(ii) $\int \chi_{r, E, X_{1} \times X_{2}} \uparrow E \mathrm{~d} \operatorname{ProdMeas}\left(M_{1}, M_{2}\right)=$ $\int \operatorname{Integral1}\left(M_{1}, \chi_{r, E, X_{1} \times X_{2}}\lceil E) \mathrm{d} M_{2}\right.$, and

(iii) $\int \chi_{r, E, X_{1} \times X_{2}} \mathrm{~d} \operatorname{ProdMeas}\left(M_{1}, M_{2}\right)=\int \operatorname{Integral} 2\left(M_{2}, \chi_{r, E, X_{1} \times X_{2}}\right) \mathrm{d} M_{1}$, and

(iv) $\int \chi_{r, E, X_{1} \times X_{2}}\left\lceil E \mathrm{~d} \operatorname{ProdMeas}\left(M_{1}, M_{2}\right)=\right.$ $\int \operatorname{Integral2}\left(M_{2}, \chi_{r, E, X_{1} \times X_{2}}\lceil E) \mathrm{d} M_{1}\right.$.

The theorem is a consequence of (1), (12), (64), (82), (78), (81), and (65).

(84) Let us consider non empty sets $X_{1}, X_{2}$, a $\sigma$-field $S_{1}$ of subsets of $X_{1}$, a $\sigma$ field $S_{2}$ of subsets of $X_{2}$, a $\sigma$-measure $M_{1}$ on $S_{1}$, a $\sigma$-measure $M_{2}$ on $S_{2}$, an element $A$ of $\sigma\left(\right.$ MeasRect $\left.\left(S_{1}, S_{2}\right)\right)$, and a partial function $f$ from $X_{1} \times$ $X_{2}$ to $\overline{\mathbb{R}}$. Suppose $M_{1}$ is $\sigma$-finite and $M_{2}$ is $\sigma$-finite and $f$ is non-negative or non-positive and $A=\operatorname{dom} f$ and $f$ is measurable on $A$. Then

(i) $\int f \mathrm{~d} \operatorname{ProdMeas}\left(M_{1}, M_{2}\right)=\int \operatorname{Integral1}\left(M_{1}, f\right) \mathrm{d} M_{2}$, and

(ii) $\int f \mathrm{~d} \operatorname{ProdMeas}\left(M_{1}, M_{2}\right)=\int \operatorname{Integral} 2\left(M_{2}, f\right) \mathrm{d} M_{1}$.

\section{REFERENCES}

[1] Grzegorz Bancerek, Czesław Byliński, Adam Grabowski, Artur Korniłowicz, Roman Matuszewski, Adam Naumowicz, Karol Pąk, and Josef Urban. Mizar: State-of-the-art and beyond. In Manfred Kerber, Jacques Carette, Cezary Kaliszyk, Florian Rabe, and Volker Sorge, editors, Intelligent Computer Mathematics, volume 9150 of Lecture Notes in Computer Science, pages 261-279. Springer International Publishing, 2015. ISBN 978-3319-20614-1. doi 10.1007/978-3-319-20615-8_17.

[2] Heinz Bauer. Measure and Integration Theory. Walter de Gruyter Inc., 2002.

[3] Vladimir Igorevich Bogachev and Maria Aparecida Soares Ruas. Measure theory, volume 1. Springer, 2007. 
[4] Noboru Endou. Product pre-measure. Formalized Mathematics, 24(1):69-79, 2016. doi:10.1515/forma-2016-0006.

[5] Noboru Endou. Fubini's theorem on measure. Formalized Mathematics, 25(1):1-29, 2017. doi:10.1515/forma-2017-0001

[6] Noboru Endou and Yasunari Shidama. Integral of measurable function. Formalized Mathematics, 14(2):53-70, 2006. doi 10.2478/v10037-006-0008-x.

[7] Noboru Endou, Katsumi Wasaki, and Yasunari Shidama. Basic properties of extended real numbers. Formalized Mathematics, 9(3):491-494, 2001.

[8] Noboru Endou, Katsumi Wasaki, and Yasunari Shidama. The measurability of extended real valued functions Formalized Mathematics, 9(3):525-529, 2001.

[9] Adam Grabowski, Artur Korniłowicz, and Adam Naumowicz. Four decades of Mizar. Journal of Automated Reasoning, 55(3):191-198, 2015. doi 10.1007/s10817-015-9345-1.

[10] P. R. Halmos. Measure Theory. Springer-Verlag, 1974.

[11] Johannes Hölzl and Armin Heller. Three chapters of measure theory in Isabelle/HOL. In Marko C. J. D. van Eekelen, Herman Geuvers, Julien Schmaltz, and Freek Wiedijk, editors, Interactive Theorem Proving (ITP 2011), volume 6898 of LNCS, pages 135-151, 2011.

[12] Yasushige Watase, Noboru Endou, and Yasunari Shidama. On $L^{1}$ space formed by real-valued partial functions. Formalized Mathematics, 16(4):361-369, 2008. doi: $10.2478 /$ v10037-008-0044-9 